\title{
Transformações quase invariantes em relação à direção de magnetização
}

Soraya Lozada Tuma, Carlos Alberto Mendonça

Instituto de Astronomia, Geofísica e Ciências Atmosféricas, IAG/USP

\section{Copyright 2004, SBGf - Sociedade Brasileira de Geofísica}

Este texto foi preparado para a apresentação no I Simpósio de Geofísica de Sociedade Brasileira de Geofísica, São Paulo, 26-28 de setembro de 2004. Seu conteúdo foi revisado pela Comissão Tecno-científica do I SR-SBGf mas não necessariamente representa a opinião da SBGf ou de seus associados. E proibida a reprodução total ou parcial deste material para propósitos comerciais sem prévia autorização da SBG.

\section{Resumo}

Este trabalho apresenta testes sintéticos de mapeamento de campos obtidos por transformações não-lineares aplicadas a anomalias magnéticas causadas por prismas tri-dimensionais. Por simulação em computador, foram identificadas transformações que produzem campos com menor grau de sensibilidade em relação à direção de magnetização e com maior capacidade de delimitação de fontes justapostas. Os resultados mostram que algumas transformações são mais eficientes que a amplitude do sinal analítico, que tem sido mais utilizada na prática.

\section{Introdução}

A interpretação de mapas magnetométricos requer processamento de dados no sentido de facilitar a delimitação das rochas magnéticas do substrato. $\mathrm{Na}$ magnetometria, a aplicação de transformações não lineares (TNLs) teve início nos anos 70 (NABIGHIAN, 1972, 1974), com a utilização da amplitude do sinal analítico (ASA). Para fontes 2-D, a ASA independe da direção de magnetização, o que permite a determinação de parâmetros relacionados à geometria das fontes mesmo desconhecendo esta propriedade física.

STAVREV \& GEROVSKA (2000) desenvolveram TNLs com o propósito de obter campos mais centrados sobre as fontes. Num aspecto mais geral, STAVREV \& GEROVSKA (2000) mostram que o sinal analítico não é a única transformação invariante (ou quase-invariante no caso 3-D) e sugerem que algumas transformações possam ser mais efetivas. Para fontes 3-D a invariância com a direção de magnetização não se verifica e as transformações apresentam diferentes graus de invariância.

Testes como modelos prismáticos isolados (STAVREV \& GEROVSKA, 2000; TUMA \& MENDONÇA, 2003) mostram que, em geral, as transformações $L, Q$ e $E$ apresentam maior capacidade de centralização dos campos sobre as fontes e menor distorção com respeito à direção de magnetização. No presente trabalho, testes são realizados com prismas justapostos e em profundidades diferentes, o que permite uma análise mais abrangente da utilidade destas transformações

\section{Transformações não lineares na magnetometria}

O termo "transformações não-lineares" não é muito difundido na magnetometria, apesar de algumas transformações utilizadas na atualidade poderem ser assim consideradas. Como exemplo, cita-se a ASA, grandeza que guarda relações de não-linearidade com respeito às fontes magnéticas (Tuma \& Mendonça, 2003).

NABIGHIAN (1972) mostrou que a ASA é uma função com forma de sino sobre a posição de contato e obteve uma solução analítica para o modelo de contato. Esta solução gerou um procedimento semi-automático para estimar a profundidade das fontes, que se aplica mesmo quando se desconhece a direção de magnetização. Certamente por este motivo, o uso da ASA experimentou grande difusão como técnica de interpretação.

ROEST et al. (1992) e HSU et al.(1996) mostraram que, embora adotando modelos 2-D, a ASA consegue delinear o contato de fontes 3-D e permite estimar a profundidade do topo. DEBEGLIA \& CORPEL (1997) usaram a ASA num modelo de dique. THURSTON \& SMITH (1997) utilizaram a fase do sinal analítico para determinar, além da profundidade do topo, o mergulho e o contraste de magnetização da interface.

Para fontes 3-D a ASA, $A \equiv|A(x, y, z)|$, foi definida (Roest et al. 1992) por

$$
A=\sqrt{\left(\frac{\partial T_{m}^{t}}{\partial x}\right)^{2}+\left(\frac{\partial T_{m}^{t}}{\partial y}\right)^{2}+\left(\frac{\partial T_{m}^{t}}{\partial z}\right)^{2}}
$$

sendo $\partial \mathrm{T}_{\mathrm{m}}^{\mathrm{t}} / \partial \mathrm{x}, \quad \partial \mathrm{T}_{\mathrm{m}}^{\mathrm{t}} / \partial \mathrm{y}$ e $\partial \mathrm{T}_{\mathrm{m}}^{\mathrm{t}} / \partial \mathrm{z}$ as derivadas direcionais da anomalia magnética, $\mathrm{T}_{\mathrm{m}}^{\mathrm{t}}$ (subscrito $\mathrm{m}$ indica direção de magnetização e sobrescrito t a componente de medida). STAVREV \& GEROVSKA (2000) propuseram TNLs genericamente denominadas T,R,L,E e $Q$. A transformação $T$ fornece o módulo do campo vetorial anômalo, $\mathrm{T}_{\mathrm{m}} \equiv\left|\overrightarrow{\mathrm{T}}_{\mathrm{m}}(\mathrm{x}, \mathrm{y}, \mathrm{z})\right|$ :

$$
T_{m}=\sqrt{\left(T_{m}^{x}\right)^{2}+\left(T_{m}^{y}\right)^{2}+\left(T_{m}^{z}\right)^{2}}
$$

sendo $T_{m}^{\mathrm{x}}, T_{\mathrm{m}}^{\mathrm{y}}, \mathrm{T}_{\mathrm{m}}^{\mathrm{z}}$ as componentes do campo vetorial $\vec{T}_{m} \cdot A$ transformação $R \equiv R(x, y, z)$ é tal que

$R=\frac{\left|T_{m}^{\mathrm{x}} \nabla \mathrm{T}_{\mathrm{m}}^{\mathrm{x}}+\mathrm{T}_{\mathrm{m}}^{\mathrm{y}} \nabla \mathrm{T}_{\mathrm{m}}^{\mathrm{y}}+\mathrm{T}_{\mathrm{m}}^{\mathrm{z}} \nabla \mathrm{T}_{\mathrm{m}}^{\mathrm{z}}\right|}{\mathrm{T}_{\mathrm{m}}}$

sendo $\nabla \mathrm{T}_{\mathrm{m}}^{\mathrm{x}}, \nabla \mathrm{T}_{\mathrm{m}}^{\mathrm{y}}, \nabla \mathrm{T}_{\mathrm{m}}^{\mathrm{z}}$ os gradientes das componentes $T_{m}^{x}, T_{m}^{y}, T_{m}^{z}$. A transformação $L \equiv L(x, y, z)$ expressa o laplaciano de $T_{m}$, ou seja $L \equiv \nabla^{2} T_{m}$, e é tal que 
$L=\frac{\left|\nabla T_{m}^{x}\right|^{2}+\left|\nabla T_{m}^{y}\right|^{2}+\left|\nabla T_{m}^{z}\right|^{2}-\left|\nabla T_{m}\right|^{2}}{T_{m}}$

A transformação $E \equiv E(x, y, z)$ é tal que

$E=\sqrt{\frac{\left|\nabla T_{m}^{x}\right|^{2}+\left|\nabla T_{m}^{y}\right|^{2}+\left|\nabla T_{m}^{z}\right|^{2}}{2}}$

e a transformação $Q \equiv Q(x, y, z)$ :

$\mathrm{Q}=\sqrt{\left|\nabla \mathrm{T}_{\mathrm{m}}^{\mathrm{x}}\right|^{2}+\left|\nabla \mathrm{T}_{\mathrm{m}}^{\mathrm{y}}\right|^{2}+\left|\nabla \mathrm{T}_{\mathrm{m}}^{\mathrm{z}}\right|^{2}-\left|\nabla \mathrm{T}_{\mathrm{m}}\right|^{2}}$

\section{Cálculo das TFNLs}

Para determinar as TFNLs nas equações (1) a (6) é necessário calcular i) as componentes $T_{m}^{\mathrm{x}}, \mathrm{T}_{\mathrm{m}}^{\mathrm{y}}, \mathrm{T}_{\mathrm{m}}^{\mathrm{z}}$; ii) as derivadas destas componentes e iii) as derivadas da anomalia magnética $\partial \mathrm{T}_{\mathrm{m}}^{\mathrm{t}} / \partial \mathrm{x}, \partial \mathrm{T}_{\mathrm{m}}^{\mathrm{t}} / \partial \mathrm{y}$ e $\partial \mathrm{T}_{\mathrm{m}}^{\mathrm{t}} / \partial \mathrm{z}$. Para modelos prismáticos, as componentes do campo vetorial podem ser calculadas a partir da expressão geral que calcula a anomalia magnética. As derivadas da anomalia magnética podem ser calculadas pelo método das diferenças-finitas ou analiticamente (TUMA \& MENDONÇA, 2003).

\section{Modelo testado}

Foi utilizado um modelo com três prismas verticais de base retangular, sendo que no teste i) a dimensão dos prismas é de $0,4 \times 2 \mathrm{~km}$, centrados numa janela que varia nas direções $x$, y de 0 a $5 \mathrm{~km}$ (Figura 1) e no teste ii) a dimensão dos prismas é de $2 \times 8 \mathrm{~km}$ centrados numa janela de $24 \times 24 \mathrm{~km}$ em ambas as direções (Figura 2) para testar a invariância das TNLs considerando efeito de interferência das fontes e variação da profundidade do topo das fontes. Os prismas têm espessura infinita, com topo na profundidade de $0,5 \mathrm{~km}$ (Figura 1) e de 2 e $3 \mathrm{~km}$ como está indicado na Figura 2; e intensidade de magnetização de $200 \mathrm{~A} / \mathrm{km}$. O campo indutor tem inclinação de $-30^{\circ}$ e declinação de $-20^{\circ}$. A declinação da magnetização foi de $-20^{\circ}$, mas a inclinação variou de $0^{\circ}$ a $-60^{\circ}$, com passo de $-15^{\circ}$ nas fontes do teste i) e apenas um dos corpos (superior esquerdo) no teste ii).

As Figuras 1 e 2 mostram os mapas dos campos transformados. De um modo geral, todas as TNLs mostram maior eficiência em centrar os campos sobre as fontes do que a anomalia magnética. Além disto, as TNLs melhor individualizam as fontes o que permite determinar o número de prismas e orientação. Para inclinação de $0^{\circ}$ (equador magnético), apenas os corpos orientados na direção Leste-Oeste são mapeados (Figura 1). Á medida que o valor da inclinação vai gradualmente para $-60^{\circ}$, o prisma na direção Norte-Sul começa a ser delimitado.

Para fontes rasas (topo em 0,5 km, Figura 1), as TNLs são em geral mais invariantes em relação à inclinação da magnetização. No intervalo de $0^{\circ}$ a $-15^{\circ}$ os corpos na direção Leste-Oeste são melhor delimitados pelas transformações $L$ e $Q$, enquanto que fontes com topo na profundidade de $3 \mathrm{~km}$ (Figura 2) são melhor percebidas pela transformação $Q$. No intervalo de $-30^{\circ}$ a $-60^{\circ}$ estas transformações melhoram seu desempenho e os campos ficam praticamente centrados sobre as fontes. No mesmo contexto, a ASA apenas apresenta melhores resultados que a anomalia magnética e a transformação $\mathrm{T}$. De um modo geral, seus resultados são inferiores aos produzidos pelas transformações $L$ e $Q$.

\section{Conclusões}

As transformações $L$ e $Q$ de (STAVREV \& GEROVSKA, 2000) são mais eficientes em delimitar as fontes prismáticas e centralizar os campo ao redor das mesmas. Isto se aplica mesmo na presença de fontes justapostas. A utilização destas transformações em mapas magnéticos (aéreos e terrestres) podem tornar mais efetivos o mapeamento das fontes e a delimitação de contatos.

\section{Agradecimentos}

Ao IAG/USP, à Embaixada do Brasil, ao Instituto Brasileiro Equatoriano de Cultura, IBEC, Quito Equador. Ao CNPQ/PEC-PG.

\section{Referências}

Debeglia, N. \& Corpel, J., 1997, Automatic 3-D interpretation of potential field data using analytic signal derivatives. Geophysics, 62: 87-96.

Hsu, S. K., Sibuet J. C. \& Shyu C. T., 1996, Highresolution detection of geologic boundaries from potentialfield anomalies: An enhanced analytic signal technique. Geophysics, 61: 373-386.

Nabighian, M. N., 1972, The analytic signal of twodimensional magnetic bodies with polygonal cross section: Its properties and use for automated anomaly interpretation. Geophysics, 37: 507-517.

Nabighian, M. N., 1974, Additional comments on the analytic signal of two-dimensional magnetic bodies with polygonal cross section. Geophysics, 39: 85-92.

Roest, W. R., Verhoef, J. \& Pilkington, M., 1992, Magnetic interpretation using the 3-D analytic signal. Geophysics, 57: 116-125.

Stavrev, P. \& Gerovska, D., 2000, Magnetic field transforms with low sensitivity to the direction of source magnetization and high centricity. Geophysical Prospecting, 48: 317-340.

Thurston, J. \& Smith, R., 1997, Automatic conversion of magnetic data to depth, dip, and susceptibility contrast using the SPI (TM) method. Geophysics, 62: 807-813.

Tuma, S., \& Mendonça, C., A., 2003, Comparação de transformações não lineares quase invariantes com a direção de magnetização. Eigth International Congress of the Brazilian Geophysical Society. 


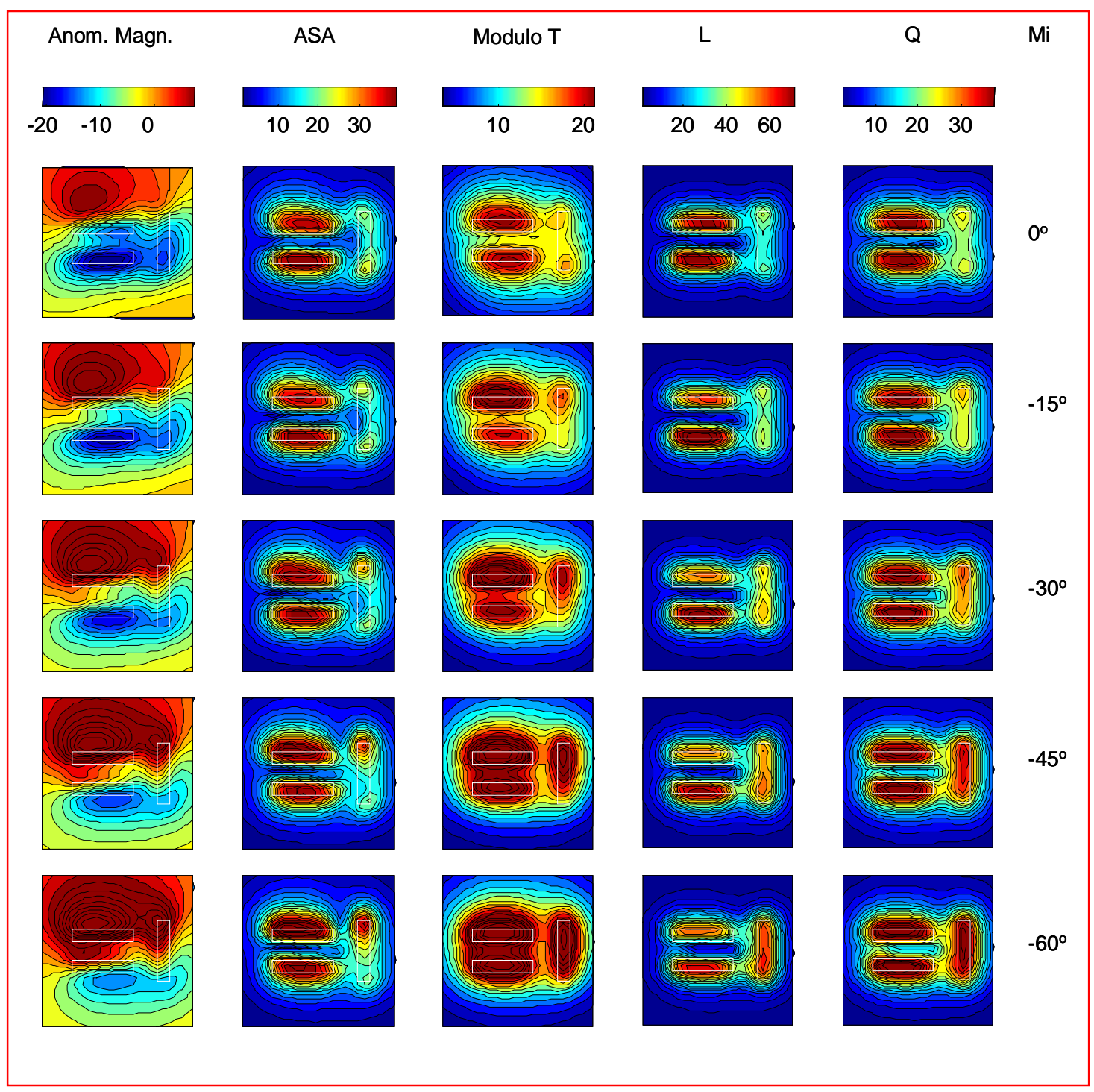

Figura 1 - Transformações do modelo de interferência de três fontes prismáticas, verticais, retangulares (em branco), topo na profundidade de $0,5 \mathrm{~km}$, campo magnético indutor externo com inclinação de $-30^{\circ}$ e declinação de -20ํㅡㄴ magnetização com declinação de $-20^{\circ}$ e inclinação variando entre $0^{\circ}$ e $-60^{\circ}$ em cada fonte. Anom. Magn.= Anomalia magnética, $A S A=A m p l i t u d e$ do sinal analítico, Módulo $T=$ Transformação $T, L=$ Transformação $L$ e $Q=$ Transformação $Q$. 


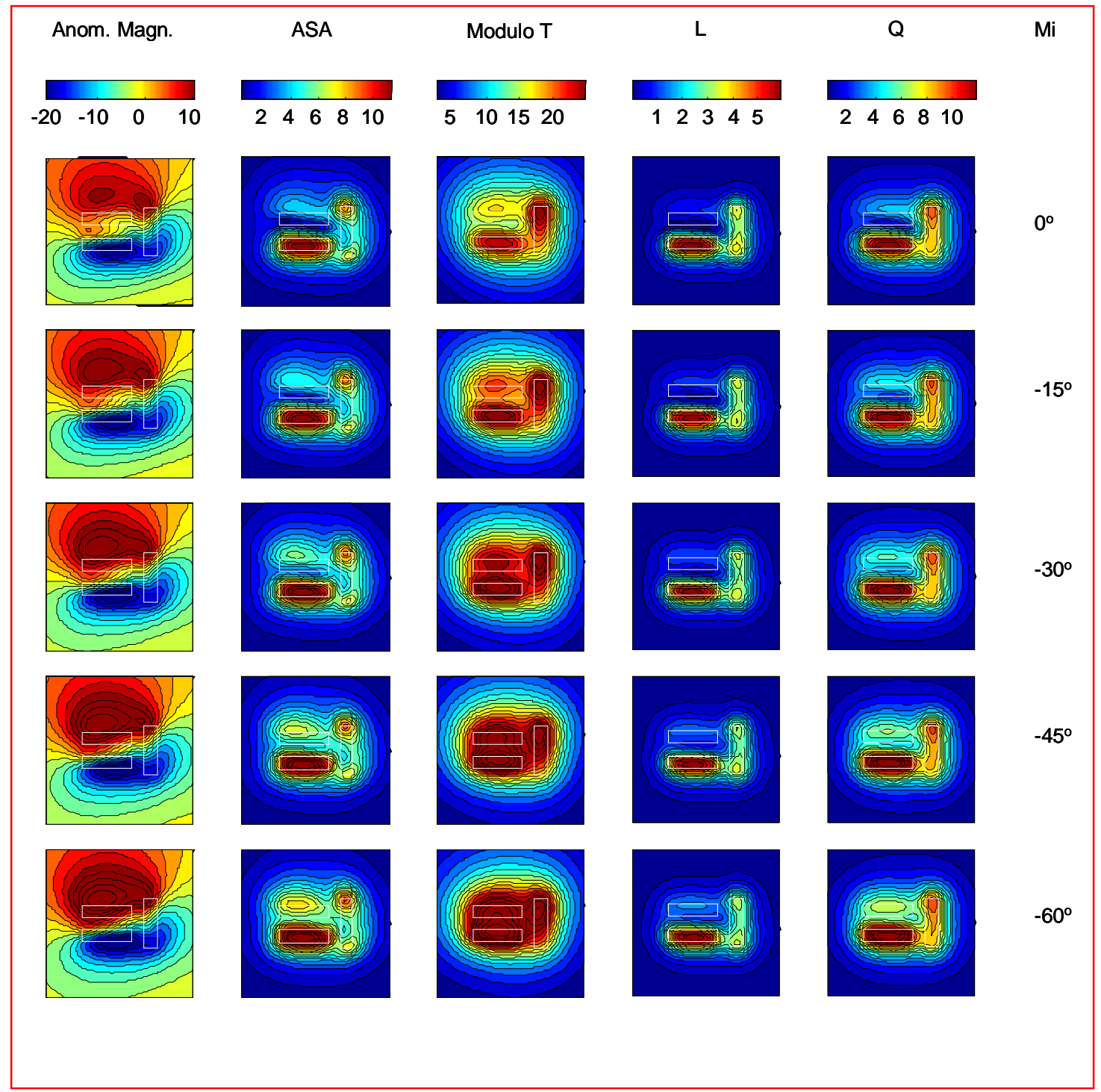

Figura 2 - Transformações do modelo de interferência de três fontes prismáticas, verticais, retangulares (em branco), topo na profundidade de $3 \mathrm{~km}$ (corpo superior esquerdo) e de $2 \mathrm{~km}$ (corpos restantes), campo magnético indutor externo com inclinação de $-30^{\circ}$ e declinação de $-20^{\circ}$, magnetização com declinação de $-20^{\circ}$ e inclinação variando entre $0^{\circ}$ e $-60^{\circ}$ apenas na fonte superior esquerda. Anom. Magn.= Anomalia magnética, ASA=Amplitude do sinal analítico, Módulo $T=$ Transformação $T, L=$ Transformação $L$ e $Q=$ Transformação $Q$. 